\title{
What Clinimetric Evidence Exists for Using Hip-specific Patient- reported Outcome Measures in Pediatric Hip Impingement?
}

\author{
Agnes G. d'Entremont PhD, Anthony P. Cooper FRCS, \\ Ashok Johari FAMS, Kishore Mulpuri MS(Ortho)
}

Published online: 4 November 2014

(C) The Association of Bone and Joint Surgeons (B) 2014

\begin{abstract}
Background Patient-reported outcomes (PROs) are an increasingly popular research tool used to evaluate the outcomes of surgical intervention. If applied appropriately, they can be useful both for disease monitoring and as a method of assessing the efficacy of treatment. Many disorders can lead to impingement in children and adolescents, but it is not clear if any PROs have been validated to evaluate outcomes in these populations.

Questions/purposes We performed a systematic review of the literature to answer the following research questions: (1) Which hip-specific PROs are used in pediatric populations with impingement? (2) What clinimetric evidence exists for the use of these specific PROs in this population?
\end{abstract}

Each author certifies that he or she, or a member of his or her immediate family, has no funding or commercial associations (eg, consultancies, stock ownership, equity interest, patent/licensing arrangements, etc) that might pose a conflict of interest in connection with the submitted article.

All ICMJE Conflict of Interest Forms for authors and Clinical Orthopaedics and Related Research ${ }^{\mathbb{R}}$ editors and board members are on file with the publication and can be viewed on request.

This work was performed at the University of British Columbia and BC Children's Hospital, Vancouver, British Columbia, Canada.

\footnotetext{
A. G. d'Entremont, K. Mulpuri

Centre for Hip Health and Mobility, University of British

Columbia, Vancouver, BC, Canada
}

A. G. d'Entremont, A. P. Cooper, K. Mulpuri

Department of Orthopaedics, University of British Columbia,

Vancouver, BC, Canada

A. G. d'Entremont, A. P. Cooper, K. Mulpuri ( $₫)$

Department of Orthopaedic Surgery, BC Children's Hospital, 1D18-4480 Oak Street, Vancouver, BC V6H 3V4, Canada

e-mail: kmulpuri@cw.bc.ca
Methods We performed two systematic searches of three databases (Medline, EMBASE, and Ovid All EBM Reviews). The first search aimed to identify specific PROs that have been applied to pediatric impingement populations. The second search aimed to find clinimetric evaluations of the PROs from the first search in this population.

Results We found six hip-specific PROs applied in pediatric impingement: Harris Hip Score, modified Harris Hip Score, Iowa Hip Score, Merle d'Aubigné Hip Score, Hip Outcome Score, and Non-arthritic Hip Score. However, we found no papers validating any of these PROs in this population. Furthermore, we found no papers validating any of these PROs in any pediatric population.

Conclusions A number of adult PROs have been applied in pediatric impingement disorders without evidence of validation in any pediatric population. Further work to develop and validate a hip-specific pediatric PRO is required.

\section{Introduction}

Femoroacetabular impingement (FAI), defined as abutment of the femoral neck on the acetabular rim or labrum, is

\author{
A. Johari \\ Children's Orthopaedic Centre, Mumbai, India
}

A. G. d'Entremont, A. P. Cooper, K. Mulpuri

Child and Family Research Institute, BC Children's Hospital,

Vancouver, BC, Canada

Present Address:

A. G. d'Entremont

Department of Mechanical Engineering, University of British

Columbia, Vancouver, BC, Canada 
associated with pain and both cartilage and labral damage. Common causes for this in children and adolescents include deformities secondary to slipped capital femoral epiphysis (SCFE) or Perthes disease. Labral damage can also occur in patients with hip dysplasia. In many cases, these disorders and resulting impingement are treated surgically, so appropriate measures for evaluating the effectiveness of surgery in these populations are important.

Patient-reported outcomes (PROs) are an increasingly popular research tool used to evaluate the outcomes of surgical intervention. They can vary from generic measures such as the SF-36 [6] to population-specific measures such as the Child Health and Illness ProfileAdolescent Edition [20] to disease- or joint- specific such as the Harris Hip Score [7]. The more specific scores focus on questions regarding the hip including pain levels, medication use, and the impact the patient's hip health has on their activity levels. Although PROs can be narrowly defined to include only measures derived entirely from patient responses, a more broad approach covering measures that combine both patient responses and clinical observations allows us to compare to previous work [12] and helps to ensure that the results include all important patient-involved measures.

The clinimetric properties of three hip-specific PROs have been investigated in adult patients with FAI [12], but it is unclear if similar clinimetric analyses have been made for pediatric impingement populations. Because of the wide range of reading ability, comprehension, and maturity in pediatric populations, it is not immediately apparent that PROs designed and validated in adult populations may be used with pediatric patients. Because PROs may be used in research studies to demonstrate treatment effectiveness, it is critical that they are shown to be valid for the populations being studied. As our understanding of FAI has increased, the application of treatment methods for this and other associated types of hip impingement has also increased. With this there has been an exponential increase in the number of publications on the subject [8] without a perceived increase in the number of PROs validated for children and adolescents. A systematic review of the use of PROs in pediatric impingement disorders provides important information to the orthopaedic community: it can confirm or refute the existence of validated measures and may uncover evidence of the quality of any validation.

Our research questions were: (1) Which hip-specific PROs are used in pediatric populations with impingement in the literature? (2) What clinimetric evidence exists for the use of these specific PROs in this population? For the purpose of this study we defined pediatric patients as those aged 18 years or younger.

\section{Search Strategy and Criteria}

\section{Literature Search}

Two separate literature searches were performed in consultation with a medical librarian. The first search was intended to identify any PROs used in a pediatric population with hip impingement disorders, and the second search was intended to identify studies that assessed the clinimetric properties of the PROs in a pediatric population with the same disorders. In the first search, we combined both subject headings and keywords for pediatric patients, for impingement and associated disorders (such as LeggCalvé-Perthes disease and SCFE), and for PROs (such as "score" or "index") (Table 1). There were no language restrictions. We used the Ovid interface to search Medline, EMBASE, and the Ovid All EBM Reviews index. We searched articles from the earliest available date in each index to December 2013.

In the second search, we combined keywords for the specific PROs found in the first search with subject headings and keywords for clinimetric evaluation ("validity", "repeatability", etc) and the same subject headings and keywords for pediatric patients and impingement and associated disorders used in the original search (Table 1).

\section{Selection Criteria}

For the first search, we included all studies of impingement and associated disorders, which applied a hip-related PRO after any surgical intervention except THA or hip resurfacing. We included studies with subjects from 0 to 18 years of age. We excluded studies with fewer than five subjects and mixed populations of adults and children and/ or adolescents.

For the second search, we included papers that evaluated clinimetric properties of the PROs found in the first search in pediatric populations with impingement and associated disorders. We excluded studies with fewer than five subjects and mixed populations of adults and children and/or adolescents.

\section{Data Abstraction}

For both searches, independent review by two authors (AGD, APC) was performed on titles and abstracts to determine which papers would undergo full-text review. Differences were resolved by consensus. Both authors also independently reviewed the full-text articles with respect to selection criteria, and differences were resolved by consensus. All articles in foreign languages were translated 
Table 1. Search term Boolean combinations for the first and second searches

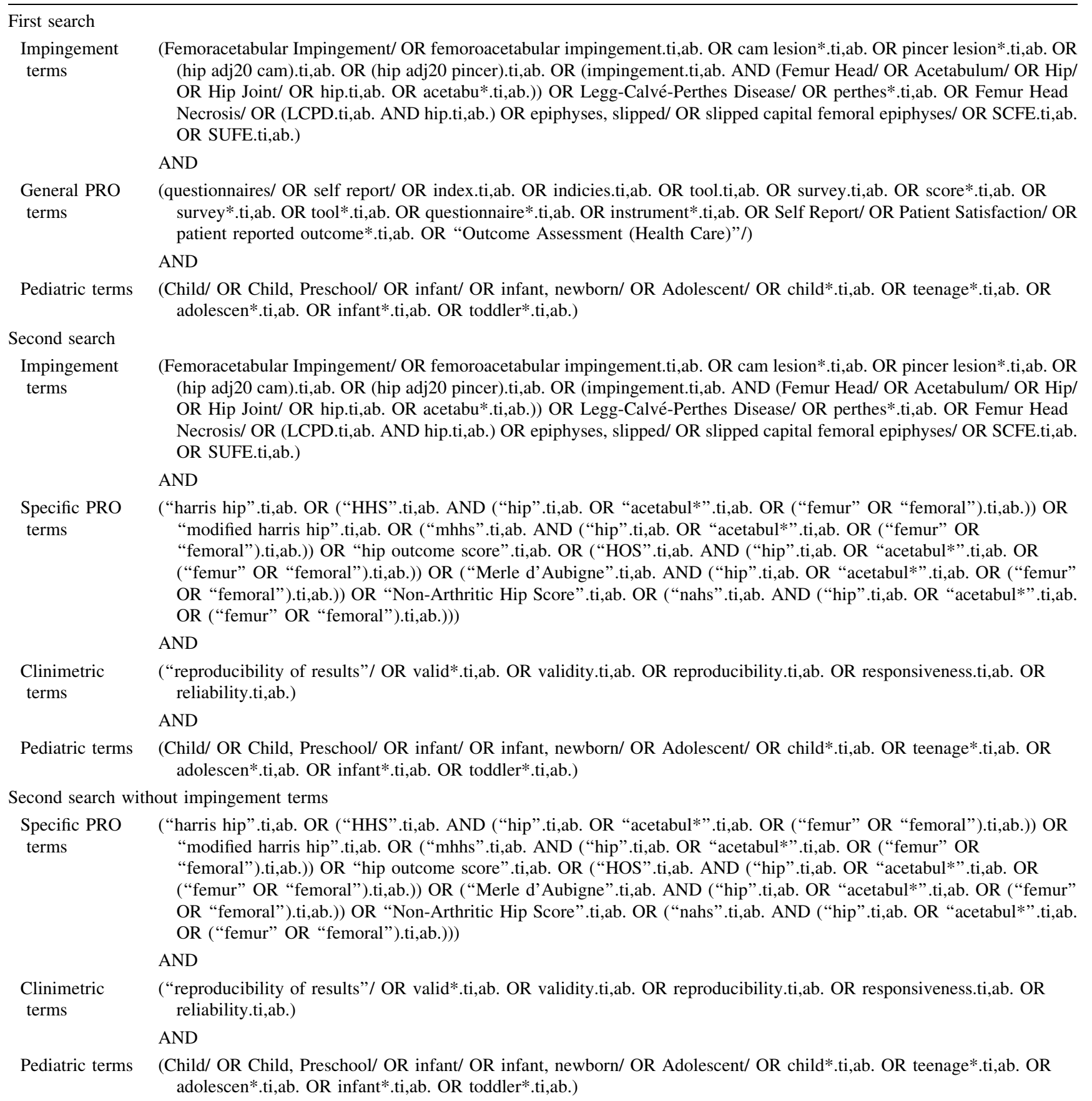

Terms followed by "/" were subject headings in Medline and were either mapped to relevant subject headings or searched as keywords in other indices depending on subject heading structures. Terms with ".ti,ab." appended were searched as keywords in both titles and abstracts. Asterisks denote wild cards, and results include all possible endings for a term (eg, child* will return child, children, and childhood). Terms with "adj20" will return results where the two words listed are used in the title or abstract within 20 words of each other; PRO = patient-reported outcome.

appropriately to determine their potential for selection. The first literature search resulted in 822 unique papers for title and abstract review. After review by two authors, 88 papers were selected for full-text review. Of these, 13 papers fit the criteria and were included to obtain relevant PROs used in these populations (Table 2; Fig. 1). The second literature search using the six PROs obtained from the first search produced 12 titles and abstracts for review. None of those papers went on to full-text review, so no papers fit the selection criteria and zero papers went on to analysis (Fig. 2A). We modified the second search to look for any papers that evaluated clinimetric properties of any of the 
Table 2. Summary of 13 studies using hip-specific PROs in pediatric populations with impingement or impingement disorders

\begin{tabular}{|c|c|c|c|c|c|c|}
\hline Study & Number & PRO & $\begin{array}{l}\text { Age at baseline } \\
\text { (mean [range]) } \\
\text { (years) }\end{array}$ & $\begin{array}{l}\text { PRO } \\
\text { preoper } \\
\text {-atively? }\end{array}$ & $\begin{array}{l}\text { Time to followup } \\
\text { (mean [range]) }\end{array}$ & $\begin{array}{l}\text { PRO at } \\
\text { followup? }\end{array}$ \\
\hline Arora et al., 2013 [3] & 26 (30 hips) & Merle d'Aubigné Score & $3(10-16)$ & No & 20 months (4-48 months) & Yes \\
\hline Dean et al., 2001 [5] & 50 (54 hips) & Harris Hip Score & $15(9-18)$ & Yes & 4 years $(2-10$ years $)$ & Yes \\
\hline Herman et al., 1996 [9] & 21 (21 hips) & Harris Hip Score & $\begin{array}{l}14 \text { (boys), } \\
12 \text { (girls) } \\
\text { (no range) }\end{array}$ & No & 3 years (no range) & Yes \\
\hline Kocher et al., 2005 [10] & 42 (48 hips) & $\begin{array}{l}\text { Modified Harris } \\
\text { Hip Score }\end{array}$ & $15(6-18$ y $11 \mathrm{~m})$ & Yes & $\begin{array}{l}17 \text { months } \\
\text { (12-26 months) }\end{array}$ & Yes \\
\hline Kumar et al., 2002 [11] & 21 (22 hips) & Harris Hip Score & $8(5-11)$ & Yes & 30 months ( $1-5$ years) & Yes \\
\hline Muratli et al., 2003 [14] & 14 (17 hips) & Iowa Hip Score & $7(5-9)$ & No & $\begin{array}{l}\text { Age at followup: } \\
12 \text { years }(10-16 \text { years })\end{array}$ & Yes \\
\hline Philippon et al., 2012 [15] & 60 (65 hips) & Modified Harris Hip Score & $15(11-16)$ & Yes & 3 years ( $2-5$ years) & Yes \\
\hline Philippon et al., 2008 [16] & 16 (17 hips) & $\begin{array}{l}\text { 1. Modified Harris } \\
\text { Hip Score } \\
\text { 2. Hip Outcome Score }\end{array}$ & $15(11-16)$ & Yes & 16 months (1-2 years) & Yes \\
\hline $\begin{array}{l}\text { Schneidmueller } \\
\text { et al., } 2006 \text { [18] }\end{array}$ & 44 (47 hips) & Merle d'Aubigné Score & $13(6-17)$ & Yes & 8 years $(1-17$ years $)$ & Yes \\
\hline Sun et al., 2011 [21] & 11 (12 hips) & Harris Hip Score & $14(9-18)$ & Yes & 6 years $(2-12$ years $)$ & Yes \\
\hline Tran et al., 2013 [23] & 34 (41 hips) & $\begin{array}{l}\text { 1. Modified Harris Hip Score } \\
\text { 2. Non-arthritic Hip Score }\end{array}$ & $16(11-18)$ & Yes & 14 months (1-2 years) & Yes \\
\hline Yoo et al., 2004 [25] & 21 (21 hips) & Iowa Hip Score & $10(6-15)$ & Yes & 7 years $(3-15$ years $)$ & Yes \\
\hline Yoo et al., 2013 [26] & 31 (31 hips) & Iowa Hip Score & $9(4-15)$ & Yes & 20 years $(15-28$ years $)$ & Yes \\
\hline
\end{tabular}

PROs $=$ patient-reported outcomes.

hip-specific PROs in any pediatric population (not limited to impingement disorders). This modified second search produced 26 papers for title and abstract review. None of these papers fit the selection criteria, resulting in no papers for analysis of clinimetric properties (Fig. 2B).

\section{Clinimetric Analysis}

The selected articles evaluating clinimetric properties of hip-specific PROs in pediatric impingement populations were to be analyzed by two authors (AGD, APC) based on the criteria by Terwee et al. [22]. Specifically, the criteria for evaluation were: content validity, internal consistency, criterion validity, construct validity, reproducibility, longitudinal validity, responsiveness, floor and ceiling effects, and interpretability.

\section{Results}

The following six hip-specific PROs were found to have been used in studies of pediatric impingement populations: (1) Harris Hip Score (HHS); (2) modified Harris Hip Score
(mHHS); (3) Iowa Hip Score; (4) Merle d'Aubigné Hip Score; (5) Hip Outcome Score (HOS) (Activities of Daily Living and Sports subscores); and (6) Non-arthritic Hip Score (NAHS) [3, 5, 9-11, 14-16, 18, 21, 23, 25, 26].

Again, we found no papers validating any of these PROs in this population. Furthermore, we found no papers validating any of these PROs in any pediatric population.

\section{Discussion}

PROs are important tools in determining the success of interventions; ideally, they should be tested for validity in each population. However, many scores are applied to new populations (such as pediatric populations) without validation. We aimed to discover which hip-specific PROs are used in pediatric populations with impingement and what clinimetric evidence exists for the use of these specific PROs in this population.

Limitations of this systematic review include our broad search for impingement and associated disorders. The lack of a concise and consistent term regularly used for hip impingement in a range of childhood disorders may have resulted in missing papers that might have been 


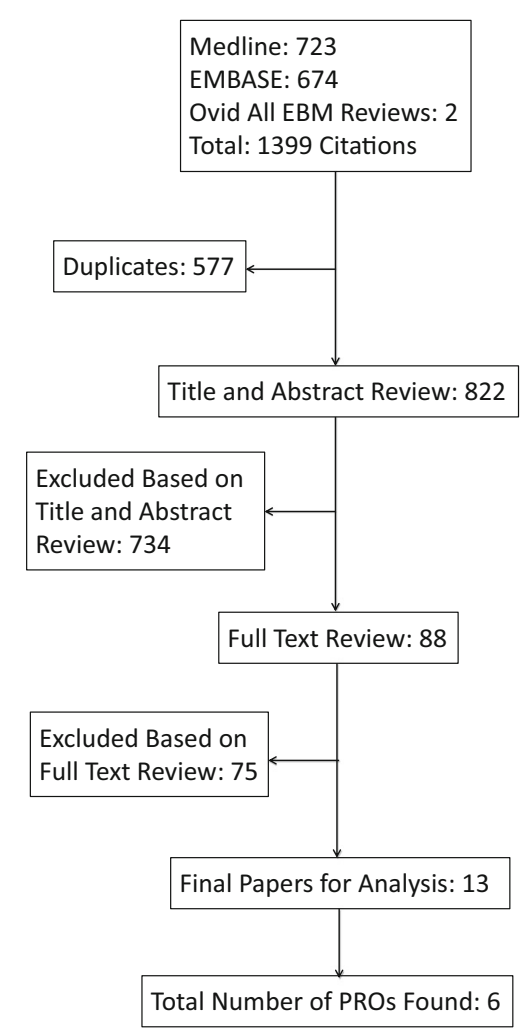

Fig. 1 The flowchart diagram shows the number citations at each stage for the first search strategy. Terms for children and adolescents, hip impingement and related disorders, and scores, surveys, and indices were used in the search (Table 1). EBM = evidence-based medicine.

included in the first search results, which might have resulted in additional PROs. However, because we performed the second search without the impingement and disorder terms, we can be more confident that we did not miss clinimetric evidence for the PROs searched in pediatric populations, including impingement. We also did not include all pediatric hip disorders in our search for measures. We restricted our search to hip impingement because this gave us the ability to focus on one disease entity with relative consistency in treatment options, namely open or arthroscopic osteoplasty. Including all hip disorders in a pediatric population would lead to results that were too heterogeneous in terms of disease processes, patient demographics, and treatment options. Another limitation is the strict inclusion of the 0 to 18 years age range and the exclusion of mixed groups of pediatric and adult subjects. The comprehension and intellectual maturity of an 18-year-old patient may not differ greatly from a 20-year-old patient; however, a choice of limit was required, because age in some excluded studies ranged from 13 up to 80 years [13]. The 0 - to 18 -year age criterion covers a broad range of both intellectual and emotional maturity and physical maturity, and we expect that is the case for the studies we found using hip-specific PROs; however, we were not able to categorize papers or PROs based on these factors, which were either unreported or unconnected to PRO outcomes. Finally, we have included instruments that combine patient-reported and clinician-reported measures (HHS, mHHS, Iowa Hip Score, Merle d'Aubigné Hip Score). Several of these scores have been identified as PROs previously in the literature [12]. Although their status as true PROs may be contentious, we chose the cautious approach of including rather than excluding any patient-based measures in a population where limited validated tools were expected.

Six hip-specific PROs were identified as having been used to study pediatric hip impingement. To our knowledge, none of these PROs were originally developed for children or adolescents. We found a slightly different range of hip-specific metrics used in pediatric impingement populations than those found in adult populations of FAI [12]. The Iowa Hip Score was not identified as having been used in adult FAI populations, and the Western Ontario and McMaster Universities Arthritis Index (WOMAC) was not identified as being used in our review of pediatric impingement populations. Otherwise, the adult PROs were the same as those used in the pediatric populations. In adult FAI, evidence of some clinimetric evaluation was found for three PROs (HOS, NAHS, and WOMAC) in FAI populations [12]. Although it did not meet our inclusion criteria as part of the first search, we found one hip- and pediatricspecific PRO called the Children's Hospital Oakland Hip Evaluation Scale (CHOHES), which is a modification of the HHS [2]. It has been validated only in a population with avascular necrosis in sickle cell disease with a mean age of 25 years (range, 8-52 years) with no data on the effect of age presented [2]. The only published application of CHOHES that we could find was in a population with osteonecrosis secondary to the treatment of developmental dysplasia of the hip [17], which is not the population it was validated in. Because of the broad inclusion of adults in the original validation, and the limited range of disorders validated, this measure does not currently fill the need for a hip- and pediatric-specific PRO for use in impingement conditions.

We found no research validating the use of six hipspecific PROs that have been applied in pediatric hip impingement, either in pediatric impingement disorders or any pediatric population. In the 13 papers found in the first search that used a hip-specific PRO in a pediatric impingement population, there was no assessment or discussion of the effect of age in the use of the PRO. None of the authors in any of the 13 papers found (six PROs) discussed the validity of applying these adult measures to children and adolescents. One study did discuss the mHHS having been developed for THA and that it has been 
Fig. 2A-B The flowchart diagram shows the number of citations at each stage for the second search strategy. Terms for the specific PROs found in the first search were combined with terms for children and adolescents and terms for clinimetric properties and used in the second search (Table 1). The first flowchart (A) shows the results for the search when we included terms for hip impingement and related disorders. The second flowchart (B) shows the search results for only the combination of specific PROs, pediatric terms, and clinimetric properties with no terms for any specific hip pathology. $\mathrm{EBM}=$ evidence-based medicine.

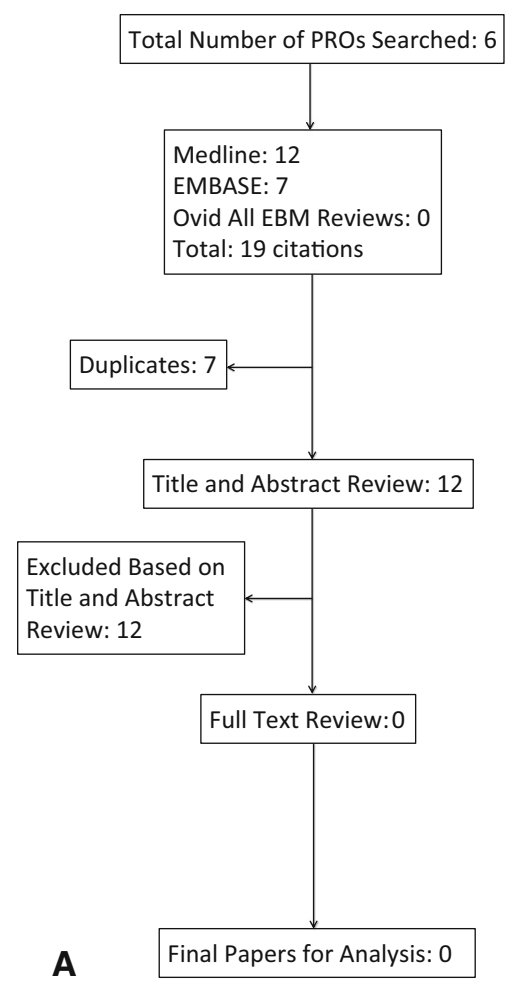

applied but not validated in hip arthroscopy [10], but again did not mention validity issues related to patient age. Despite including children who were younger than 7 years old $[10,11,18,25,26]$ in five studies using four of the six PROs found (mHHS, HHS, Iowa, Merle d'Aubigné), the papers reviewed indicated no issues in the administration of these adult PROs (Table 2). Several papers directly compared results from a PRO administered to pediatric patients with results from adult studies using the same PRO $[5,10,16,23,26]$. It is clear that a hip-specific PRO validated for pediatric patients in a range of conditions, including impingement, is needed to fill a gap in the range of outcome measures available to clinicians and researchers. There are challenges in designing PROs for a pediatric population (the child's understanding of questions, understanding of their own disease, variations with age and development [4]); however, it has been shown that children as young as 5 years old can reliably and validly self-report their health-related quality of life when asked to complete an age-appropriate instrument, achieving both internal consistency and external reliability (comparable to adolescents) [24]. In contrast, the use of proxy reporting by parents or caregivers has been shown to be unreliable [1, 19]. Appropriately developed and validated scores would give these patients the ability to consistently and reliably report their perspectives on their hip health. It is also clear that education in appropriate use of hip-specific PROs in research is needed so that researchers gain awareness in the limits of validity of the instruments that are applied in studies.

In this systematic review, we found six hip-specific PROs that have been applied to pediatric impingement populations but were unable to find any research validating these PROs in any pediatric population. This has significant implications on the validity of the outcomes reported in studies using these tools. Given that the primary aim of surgery for an impingement disorder is to improve the quality of life for these patients, the lack of validation in outcome measures may limit the validity of conclusions based on these outcome scores and the ability to compare outcomes between different studies. As such, further work is required to develop and validate a hipspecific PRO for children with impingement and associated disorders.

Acknowledgments We thank Tricia Yu (Medical Liaison Librarian, University of British Columbia) for her advice in developing our search strategies.

\section{References}

1. Achenbach TM, McConaughy SH, Howell CT. Child/adolescent behavioral and emotional problems: implications of cross-informant correlations for situational specificity. Psychol Bull. 1987;101:213.

2. Aguilar CM, Neumayr LD, Eggleston BE, Earles AN, Robertson SM, Jergesen HE, Stulberg BN, Vichinsky EP. Clinical 
Evaluation of avascular necrosis in patients with sickle cell disease: Children's Hospital Oakland Hip Evaluation Scale-a modification of the Harris hip score. Arch Phys Med Rehabil. 2005;86:1369-1375.

3. Arora S, Dutt V, Palocaren T, Madhuri V. Slipped upper femoral epiphysis: outcome after in situ fixation and capital realignment technique. Indian J Orthop. 2013;47:264-271.

4. Connolly MA, Johnson JA. Measuring quality of life in paediatric patients. Pharmacoeconomics. 1999;16:605-625.

5. Dean GS, Kime RC, Fitch RD, Gunneson E, Urbaniak JR. Treatment of osteonecrosis in the hip of pediatric patients by free vascularized fibular graft. Clin Orthop Relat Res. 2001;386:106113.

6. Garratt AM, Ruta DA, Abdalla MI, Buckingham JK, Russell IT. The SF 36 health survey questionnaire: an outcome measure suitable for routine use within the NHS? BMJ. 1993;306:14401444.

7. Harris WH. Traumatic arthritis of the hip after dislocation and acetabular fractures: treatment by mold arthroplasty. An endresult study using a new method of result evaluation. J Bone Joint Surg Am. 1969;51:737-755.

8. Haviv B, Burg A, Velkes S, Salai M, Dudkiewicz I. Trends in femoroacetabular impingement research over 11 years. Orthopedics. 2011;34:e29-e32.

9. Herman MJ, Dormans JP, Davidson RS, Drummond DS, Gregg JR. Screw fixation of grade III slipped capital femoral epiphysis. Clin Orthop Relat Res. 1996;322:77-85.

10. Kocher MS, Kim Y, Millis MB, Mandiga R, Siparsky P, Micheli LJ, Kasser JR. Hip arthroscopy in children and adolescents. $J$ Pediatr Orthop. 2005;25:680-686.

11. Kumar D, Bache CE, O'Hara JN. Interlocking triple pelvic osteotomy in severe Legg-Calvé-Perthes disease. J Pediatr Orthop. 2002;22:464-470.

12. Lodhia P, Slobogean GP, Noonan VK, Gilbart MK. Patientreported outcome instruments for femoroacetabular impingement and hip labral pathology: a systematic review of the clinimetric evidence. Arthroscopy. 2011;27:279-286.

13. Martin RL, Philippon MJ. Evidence of reliability and responsiveness for the hip outcome score. Arthroscopy. 2008;24:676682.

14. Muratli HH, Can M, Yagmurlu MF, Aktekin CN, Bicimogllu A, Tabak AY. The results of acetabular shelf procedures in LeggCalve-Perthes disease. Acta Orthop Traumatol Turc. 2003;37: 138-143.
15. Philippon MJ, Ejnisman L, Ellis HB, Briggs KK. Outcomes 2 to 5 years following hip arthroscopy for femoroacetabular impingement in the patient aged 11 to 16 years. Arthroscopy. 2012;28:1255-1261.

16. Philippon MJ, Yen Y, Briggs KK, Kuppersmith DA, Maxwell RB. Early outcomes after hip arthroscopy for femoroacetabular impingement in the athletic adolescent patient. J Pediatr Orthop. 2008;28:705-710.

17. Roposch A, Liu LQ, Offiah AC, Wedge JH. Functional outcomes in children with osteonecrosis secondary to treatment of developmental dysplasia of the hip. J Bone Joint Surg Am. 2011;93:e145.

18. Schneidmueller D, Carstens C, Thomsen M. Surgical treatment of overgrowth of the greater trochanter in children and adolescents. J Pediatr Orthop. 2006;26:486-490.

19. Sprangers MA, Aaronson NK. The role of health care providers and significant others in evaluating the quality of life of patients with chronic disease: a review. J Clin Epidemiol. 1992;45:743760 .

20. Starfield B, Ensminger M, Riley A, Mcgauhey P, Skinner A, Kim S, Bergner M, Ryan S, Green B. Adolescent health status measurement: development of the child health and illness profile. Pediatrics. 1993;91:430-435.

21. Sun W, Li Z-R, Shi Z-C, Yang Y-R, Wang B-L, Guo W-S, Liu ZH. Early and middle term results after surgical treatment for slipped capital femoral epiphysis. Orthop Surg. 2011;3:22-27.

22. Terwee CB, Bot SD, de Boer MR, van der Windt DA, Knol DL, Dekker J, Bouter LM, de Vet HC. Quality criteria were proposed for measurement properties of health status questionnaires. J Clin Epidemiol. 2007;60:34-42.

23. Tran P, Pritchard M, O'Donnell J. Outcome of arthroscopic treatment for cam type femoroacetabular impingement in adolescents. ANZ J Surg. 2013;83:382-386.

24. Varni JW, Limbers CA, Burwinkle TM. How young can children reliably and validly self-report their health-related quality of life? An analysis of 8,591 children across age subgroups with the PedsQL 4.0 Generic Core Scales. Health Qual Life Outcomes. 2007;5:1.

25. Yoo WJ, Choi IH, Chung CY, Cho TJ, Kim HY. Valgus femoral osteotomy for hinge abduction in Perthes' disease. J Bone Joint Surg Br. 2004;86:726-730.

26. Yoo WJ, Choi IH, Moon HJ, Chang S. Valgus Femoral osteotomy for noncontainable Perthes hips: prognostic factors of remodeling. J Pediatr Orthop. 2013;33:650-655. 